\title{
La contextualisation de la théologie protestante comme lieu de changement du christianisme en Océanie
}

The contextualisation of protestant theology in Oceania as a Sign of a Changing Christianity

La contextualización de la teología protestante como lugar de cambio del cristianismo en el Pacífico Sur

\section{Gilles Vidal}

\section{(2) OpenEdition}

Journals

Édition électronique

URL : http://journals.openedition.org/assr/23667

DOI : 10.4000 /assr.23667

ISSN : $1777-5825$

\section{Éditeur}

Éditions de l'EHESS

Édition imprimée

Date de publication : 1 avril 2012

Pagination : 157-177

ISBN : 978-2-7132-2328-0

ISSN : 0335-5985

\section{Référence électronique}

Gilles Vidal, « La contextualisation de la théologie protestante comme lieu de changement du christianisme en Océanie ", Archives de sciences sociales des religions [En ligne], 157 | janvier-mars 2012, mis en ligne le 26 avril 2012, consulté le 19 mars 2021. URL : http://journals.openedition.org/ assr/23667 ; DOI : https://doi.org/10.4000/assr.23667 


\section{Gilles Vidal}

\section{La contextualisation de la théologie protestante comme lieu de changement du christianisme en Océanie}

Compte tenu de l'importance historique et sociologique du christianisme en Océanie, le discours théologique constitue l'un des lieux d'observation privilégiés du champ social. Notre propos est ici de décrire l'évolution de ce discours religieux dans sa version protestante. Au niveau épistémologique, nous n'aborderons pas la théologie d'un point de vue normatif. Nous entendons simplement retracer, sur un plan sociohistorique, les origines et les filiations des concepts utilisés par certains théologiens océaniens contemporains, en nous inscrivant dans une approche dialogique entre théologie, anthropologie et histoire, élaborée au début du XX ${ }^{\mathrm{e}}$ siècle par $\mathrm{M}$. Leenhardt sous le terme de missiologie descriptive et pratiquée depuis en Europe du Nord et en milieu anglo-américain (Zorn, 2004).

La démarche consistant à organiser une réflexion théologique à partir de la culture est généralement désignée dans la littérature missiologique en milieu protestant sous le vocable de « contextualisation » ou "d'inculturation » en milieu catholique romain (Vidal, 2011 : 341-358). Ce concept articule la notion théologique de révélation et l'idée anthropologique d'acculturation. Il a été systématisé, dans les années 1960, au sein du Conseil CEcuménique des Églises (COE) en ces termes : " ... la contextualisation authentique (...) surgit d'une rencontre vraie et profonde entre la Parole de Dieu et le monde dans lequel elle prend place ; elle est déterminée par la volonté de mettre au défi et de transformer une situation donnée, en s'enracinant dans, et en participant à un temps historique précis. Il est donc clair que la contextualisation est un processus dynamique, non un processus statique. Elle constate que toute situation humaine évolue sans arrêt et elle reconnaît la possibilité d'un vrai changement; en cela, elle ouvre le chemin pour l'avenir» (Blaser, 1995 : 134-135).

Afin d'étudier l'émergence et l'évolution de la théologie protestante océanienne contemporaine, nous avons sélectionné trois théologiens que l'on peut considérer comme des "figures de proue » des années 1970 jusqu'aux années 2000 : Sione Amanaki Havea, de Tonga, Ilaitia Sevati Tuwere, de Fidji et Ama 
Amalele Tofaeono, de Samoa. Tous trois proviennent d'Églises issues directement des anciennes Sociétés des Missions à l'œuvre depuis le XVIII ${ }^{\mathrm{e}}$ siècle. Lorsque nous évoquons ici la théologie protestante océanienne, nous n'incluons pas la production d'autres Églises de la mouvance évangélique, pentecôtiste, ou relevant de "nouveaux mouvements religieux » présents dans la Région (Ernst, 2006).

Nous présenterons successivement la biographie de chacun des théologiens précités ainsi que l'un des thèmes majeurs de leur pensée. Dans un deuxième temps, nous tenterons de cerner les continuités et les ruptures de ce discours religieux particulier ainsi que les influences qu'il a subies. Enfin nous proposerons pour conclure une périodisation historique de la théologie protestante en Océanie à partir de sa mise en regard avec le christianisme régional et mondial et ses évolutions.

\section{Éléments du discours théologique dans le Pacifique}

\section{La communauté chez S. A. Havea}

La théologie protestante contemporaine dans le Pacifique insulaire ne prend véritablement son essor qu'au début des années 1960, lors de la création de la Conférence des Églises du Pacifique ${ }^{1}$ initiée par la conférence de Malua (Samoa) en 1961 et l'inauguration du Pacific College of Theology (PTC) en 1966 à Suva, c'est-à-dire au moment de ce que J. Barker appelle la deuxième vague de la christianisation en Océanie.

Une figure théologique se détache de cette époque : Sione Havea, né sur l'île de Vava'u à Tonga en 1922 dans une famille de pasteurs, considéré comme le père fondateur de la théologie du Pacifique. Après des études secondaires, il entre en 1940 au Collège Tupou, institut de formation théologique. En 1941 et 1942, il participe à une tournée d'évangélisation en Australie organisée par la Methodist Missionary Society (MMS) avec le pasteur fidjien Setareki A. Tuilovoni. De 1952 à 1955, Havea suit des études de théologie à l'Université de Drew dans le New Jersey où il obtient une licence en théologie (Bachelor of Divinity). Délégué officiel de l'Église méthodiste en Australasie il participe à la deuxième Assemblée mondiale du COE à Evanston. De retour à Tonga, il commence une carrière d'enseignement, au Collège Tupou puis, en 1968 comme directeur du Collège Théologique de Sia'atoutai. En 1958, l'Université de Tokyo lui décerne le titre de Docteur Honoris Causa pour son ministère d'enseignant et de pasteur. Parallèlement, il est élu secrétaire général de la Free Wesleyan Church of Tonga avant d'en devenir

1. Pacific Conference of Churches (PCC). 
le président de 1971 à 1977. Il assume également la fonction de chapelain du roi Tupou IV. À la même époque, de 1971-1976, il est élu au Bureau du Conseil Méthodiste Mondial (World Methodist Council). En 1977, il est proposé comme doyen du PTC à Fidji, poste qu'il occupera jusqu'en 1982. Il fait aussi partie de la délégation de la PCC à la $6^{\mathrm{e}}$ assemblée mondiale du COE à Vancouver en 1983. Son épouse Etina décède un an seulement après l'arrivée du couple à Fidji ${ }^{2}$.

Havea se retire ensuite de la scène théologique régionale mais reste engagé à Tonga comme président de son Église de 1982 à 1992, année de sa retraite. Havea a joué un rôle sinon prophétique, du moins précurseur dans presque toutes les fonctions qu'il a occupées : premier président élu de son Église, premier directeur tongien du Collège Sia'atoutai, premier président du Conseil National des Églises de Tonga, premier directeur océanien du PTC, etc. Cette fonction de pionnier au niveau institutionnel national et régional renforce le crédit attaché à ses positions théologiques parmi lesquelles la vie communautaire joue un rôle fondamental.

Havea offre en effet une place de choix aux valeurs communautaires océaniennes, rendues visibles en particulier lors des grandes cérémonies coutumières. Il prône ainsi une théologie de la célébration et du don : "Notre théologie du Pacifique pourrait être une théologie de célébration. La participation de la communauté, le caractère inclusif de la famille élargie, le partage et le soin que nous prenons des anciens étaient des caractéristiques des peuples du Pacifique. (...) Appliquons maintenant cela à la théologie. Dieu, dans sa célébration du don (l'eucharistie) fit le premier geste en donnant son saint Fils. Les gens répondent à ce geste en faisant à leur tour des dons d'argent, de nourriture, de fleurs, qui les font participer à une célébration entre Dieu et la communauté. Dieu fit le premier pas. Il initia le don et les gens furent nourris de louanges et d'Actions de grâces. » (Havea, $1987:$ 13).

Quant à la question des destinataires d'une théologie du Pacifique, une expression phare ressort de tous ses écrits : "le peuple » en tant qu'acteur de la culture. Dans ce sens, le Pacifique se démarque clairement de l'Amérique latine des années 1970 où la notion de peuple, également utilisée dans la théologie de la libération, était fortement connotée par un arrière-plan politique d'un gouvernement par le prolétariat. Rien de tel chez Havea le Tongien dont la société est caractérisée par une sacralisation exacerbée de la monarchie!

Contrairement donc à la théologie de la libération Sud-américaine fondée sur un raisonnement de type marxiste, la théologie pour le peuple de Havea repose sur les valeurs culturelles qu'il considère comme intrinsèques du Pacifique. Elles s'expriment en termes de rassemblement de la communauté, de partage et de

2. Le Centre de formation pratique et théologique des femmes du PTC porte aujourd'hui son nom. 
solidarité, voire de guérison. Elles se fondent sur les devoirs qui conditionnent la vie en société. La communauté des pauvres est ici assimilée au peuple de Dieu et la mission de l'Église, qui fait partie de cette même communauté, doit être celle du service de tous. Loin d'intégrer la culture dans une théologie préconstruite, Havea renverse la perspective en recourant à une herméneutique du symbole : penser Dieu à partir de la communauté humaine et de ses valeurs propres. Telle est selon lui la tâche de la contextualisation à entreprendre pour une théologie pertinente.

\section{Le Vanua chez Sevati Tuwere}

Sevati Tuwere se caractérise quant à lui non seulement par son enseignement théologique mais aussi par son rôle éminent dans l'histoire récente de son pays. Né en 1940 sur l'île de Vanua Levu à Fidji, il fait partie de la première génération des étudiants formés au PTC où il obtient sa licence (Bachelor of Divinity) en 1969. Il complète sa formation universitaire en 1989 par une maîtrise au Melbourne College of Divinity, puis par un doctorat en Théologie. Après avoir été directeur au Davuilevu Theological College de Fiji, il succède à Sione A. Havea comme Principal du PTC de 1982 à 1988. Il y retourne en 1993 pour y enseigner la théologie systématique avant d'être d'abord président, puis secrétaire général de l'Église méthodiste à Fiji et Rotuma jusqu'en 2000. Délégué de son Église, il participe activement au mouvement œcuménique régional et mondial. Il quitte Fidji en 2001 pour le Collège St John à Auckland où il enseigne la théologie systématique.

Cependant cette brève biographie académique et ecclésiale ne rend pas justice au rôle fondamental joué par Tuwere dans l'histoire contemporaine des Fidji. Comme secrétaire général de l'Église, il a en effet été au premier plan des événements survenus entre mai et juillet 2000. Cette année-là, pour la première fois depuis l'indépendance du pays en 1970, un Premier Ministre d'origine indienne, Mahendra Chaudhry, venait d'être nommé à la tête d'un gouvernement travailliste. Ressentant cela comme une atteinte à l'identité et la souveraineté fidjienne, Georges Speight, un homme d'affaires fidjien appuyé par des nationalistes de sa région - dont un certain nombre de chefs traditionnels et de pasteurs méthodistes prend le gouvernement en otage dans les locaux du Parlement pendant trois mois, demandant l'abrogation pure et simple du gouvernement et de nouvelles élections, ce qui sera finalement obtenu par la suite ${ }^{3}$.

Au cours de ces trois mois, au nom de l'Église Méthodiste, Tuwere a notamment organisé une marche pacifique réclamant la libération des otages. Agissant en pasteur, il se rend plusieurs fois à l'intérieur du Parlement, pour célébrer des cultes et des prières de soutien, réconfortant aussi bien les otages que certains

3. Voir sur ce point Tuwere, 1993. 
de leurs geôliers, tandis qu'à l'extérieur, d'autres pasteurs, parfois de la même Église, mais le plus souvent appartenant à des groupes religieux fondamentalistes prêchaient pour la cause putschiste (Field : 2005). Lors de cette crise, Tuwere réussit à faire prendre à l'Église méthodiste une motion officielle condamnant la prise d'otage. Ceci relevait de l'exploit dans la mesure où l'Église, déjà très impliquée dans le passé lors du coup d'État du lieutenant-colonel Rabuka en 1987, par ailleurs prédicateur dans l'Église Méthodiste, était profondément divisée sur la question. En raison des solidarités familiales traditionnelles, un grand nombre de membres de l’Église Méthodiste - première force religieuse du pays - s'est retrouvée plus ou moins contraint de soutenir ce putsch et de fournir une aide logistique aux preneurs d'otage. Le président de l'Église d'alors, Tomasi Kanailagi, ancien camarade d'étude au PTC de Tuwere, était d'ailleurs ouvertement opposé à la motion de son secrétaire général, n'hésitant pas à publier une sorte de contre motion "à titre privé » exprimant son soutien aux revendications des amis de G. Speight, tout en sachant qu'en sa qualité de président, elle aurait de facto un statut quasi officiel...

Ces deux positions inconciliables, incarnées par les deux dirigeants principaux de la même Église, au milieu des oppositions ethniques entre Indo-Fidjiens et Fidjiens « de souche » forment l'arrière-plan de la théologie de Tuwere. Toute sa quête - ancrée dans son existence et son expérience de dirigeant d'Église au sein de ce contexte-là - est sous-tendue par cette profonde déchirure où « l'autre » n'est le plus souvent perçu que comme une menace, un individu à soumettre sur une terre qui n'est pas la sienne. C'est au cœur de ce débat nationaliste qu'il faut replacer le concept central de vanua que va développer Tuwere.

Dans son ouvrage majeur issu de sa thèse de doctorat intitulé Vanua: towards a fijian Theology of place, il désigne lui-même sa théologie comme " une théologie du lieu ». Il définit le vanua ainsi : "Dans un bon nombre de langues du Pacifique, l'expression "sein maternel" 4 désigne aussi la "terre" 5 . Les meilleurs exemples sont : fanua (Samoa), fonua (Tonga), fenua (Maohi Nui ou Tahiti). On trouve des parallèles dans d'autres endroits : vanua (Fidji) et lau fanua (Tuvalu). Ces mots ont la même caractéristique commune et se rapportent aux montagnes, rivières, vallées, plantes, arbres et pratiquement tout ce qui se trouve à la surface de la terre incluant le sol ou la terre elle-même. Mais ils renvoient également au-delà de ces éléments matériels en se référant aux gens, à l'identité, aux coutumes, traditions, ancêtres, croyances et valeurs » (Tuwere, 1989:10).

\footnotetext{
4. En anglais «womb», litt. «utérus ».

5. Ou le «pays », « land» en anglais. Nous abordons ici une difficulté de traduction importante : faut-il parler de "pays » avec une connotation relevant plutôt de la géographie humaine ou de "terre " dans l'optique d'une géographie physique ? En choisissant l'une ou l'autre traduction on risque de donner à la théologie de Tuwere soit une connotation ethnique soit une connotation politique.
} 
Le vanua correspond donc grosso modo au "pays " au sens où ce mot à l'avantage de réconcilier la géographie physique et la géographie humaine: le pays et tout ce qu'il contient, tout ce qui le constitue non comme nation dans une acception politique, mais comme le home ou homeland anglais. Trois concepts majeurs forment l'armature de sa théologie : le pays, la Trinité et la création.

Le vanua est tout d'abord le pays, lieu historique de la révélation : "force de cohésion sociale » mais aussi lieu de conjonction du présent, du passé et de l'avenir, de l'humanité et du reste de la création, du monde visible et du monde invisible. La valeur spirituelle du vanua doit être pleinement reconnue et non simplement sa valeur marchande ou sentimentale.

Cependant, la dimension temporelle de ce vanua est elle aussi affirmée : le vanua n'est pas devenu le lieu de la révélation, mais il l'était déjà du fait de son statut de création. Le lotu - le christianisme ou la religion chrétienne - n'a fait en quelque sorte " que révéler la révélation » en offrant une nouvelle interprétation de la relation de l'homme à Dieu. Exprimée ainsi sous une forme restrictive, cette thèse pourrait laisser croire à une minimisation du rôle du lotu et de l'Évangile, or il n'en est rien puisque tous deux doivent garder une fonction critique essentielle vis-à-vis de ses deux autres partenaires de triade vanua et matanitu (le pouvoir temporel). Chacun, dans ce dispositif tripartite jouant un rôle d'instance critique à l'égard des deux autres.

En fait, la nouveauté de la théologie du vanua réside précisément dans son ancrage historique, alors que la problématique du lieu, de la terre et de l'appartenance pourrait très facilement la confiner à une théologie de type particulariste ou « régionale ». Or il est question ni de s’isoler du reste du monde, ni de « périodiser " l'histoire du salut en deux ères, celle des ténèbres (ère pré-missionnaire) et celle de la lumière (arrivée de l'Évangile) : "Il ne peut y avoir deux histoires dans l'économie du salut de Dieu : l'histoire des missionnaires et une histoire "sombre" de la culture réceptrice. Il n'y a qu'une histoire, l'histoire du salut dans laquelle le Dieu trinitaire est continuellement à l'œuvre, dont le centre et la force centrifuge est Jésus le Christ de Dieu. L'histoire de la terre et de la mer qui inclut nos mythes, nos croyances et nos systèmes de valeurs fait partie de l'histoire du salut» (Tuwere, $1995: 11$ ).

Tuwere rejoint ici le concept d'ephapax ${ }^{6}$ cher à Havea : une révélation à la fois historique et géographique. Il reprend également la critique de ce dernier sur l'écriture de l'histoire par les acteurs européens : " les acteurs locaux remplissant les notes de bas de page (...) La théologie de l'histoire doit concerner l'oikuménè, l'ensemble de ce qui est habité, la planète, la terre et la mer. (...) Il doit y avoir un lien direct entre l'Océanie et Israël, et non via Sydney, Londres ou Rome » (Tuwere, 1992 : 51-52).

6. Litt. "une fois pour toutes ». Cette expression dénote le caractère fondamentalement unique d'un événement, ici la Révélation. 
Le théologien fidjien recourt ensuite à La Trinité comme modèle de conceptualisation des relations à l'intérieur de la triade vanua/lotu/matanitu. On voit ici se développer une voie que Havea n'avait jusque-là fait qu'esquisser. Comme nous le verrons plus bas, il puise cette inspiration sur La Trinité dans le mouvement œcuménique et plus fondamentalement chez le théologien allemand Jürgen Moltmann qu'il cite abondamment ${ }^{7}$. Ce locus théologique classique est envisagé à partir de deux axes majeurs : le christocentrisme et l'éthique sociale.

La christologie prédomine nettement à l'intérieur même du trinitarisme affiché de l'auteur. La réhabilitation d'une création «bonne » ou la valorisation de la culture comme lieu de la révélation ne doivent pas occulter ce christocentrisme. La partie centrale de son ouvrage majeur, Vanua, porte sur le Christ comme image ou interface entre Dieu et le pays. Or celui-ci ne peut se comprendre qu'en fonction d'un premier lieu, déjà donné et en même temps ultime, la croix de Jésus : "Y a-t-il une norme chrétienne selon laquelle nous devrions vivre ? Oui, je crois qu'il y en a une et c'est la croix du Christ. Voilà le seul point décisif pour distinguer ce qui est chrétien de ce qui ne l'est pas » (Tuwere, 1994 : 29).

Tuwere entend appliquer à l'éthique sociale la doctrine théologique de la Trinité qu'il emprunte à Moltmann. C'est parce que la relation dite classiquement ad intra des personnes de la Trinité se fonde sur l'amour et la compassion, que ce même amour peut rejaillir ad extra, c'est-à-dire dans la relation entre ces personnes et le monde. Le Père, le Fils et le Saint-Esprit servent de «paradigmes théologiques » car ils sont "des principes à la fois intégrateurs et critiques » (Tuwere, 2002 : 17). La Trinité opère donc comme archétype de l'unité dans la diversité, comme "base du pluralisme permettant de la différentiation mais sans séparation » (Tuwere, 1997 : 86). Pour le théologien fidjien ce schéma relationnel, analogue aux valeurs traditionnelles fidjiennes telles que l'écoute, présente un caractère heuristique capital dans une situation politique et ethnique aussi tendue soit-elle. Il vise une " anthropologie théologique » destinée à la délivrance d'une orientation éthique pour son propre peuple.

On ne peut comprendre cette posture théologique en dehors de la situation existentielle de l'auteur qui la conditionne si fortement. D'un dogme de la Trinité pouvant si facilement servir de justification à une théologie de cabinet, Tuwere parvient à tirer une légitimation pour une théologie de la vie, pratique, faite de réconciliation et de justice sociale. Ces deux derniers points comme éléments constitutifs du vanua ressortent très clairement de l'étude de J. Ryle sur la cérémonie de pardon - bulubulu - organisée à la mémoire du révérend Thomas Baker en 2003 : il est le « lieu du pardon à la fois horizontal et vertical ».

7. Pas moins de cinq ouvrages dans la bibliographie de Vanua, towards a Fidjian Theology of Place. Jürgen Moltmann (1926-) est un théologien allemand de tradition réformée développant une théologie politique, puis à partir des années 1980, une théologie écologique qui influence fortement le COE. L'une des marques de sa pensée est de se situer dans un cadre trinitaire à l'intérieur duquel se joue la dynamique de la divinité. 
Enfin, le troisième lieu théologique important pour l'auteur concerne la création et son articulation avec la rédemption. Loin de renier son héritage méthodiste, Tuwere est conscient du danger de présenter cette articulation sous la forme d'une alternative ruineuse. La notion fondamentale d'appartenance de l'humain au vanua permet au théologien fidjien de dessiner les contours de l'articulation entre création et rédemption en réhabilitant une conception holistique du salut. Sa sotériologie ${ }^{8}$ s'appuie sur « l'ici et maintenant " de la présence du matanivanua, la "face du pays ", assimilée au Christ, laissant entendre par là que la rédemption se trouve au cœur même de la création et non en concurrence avec elle. Ce thème fondamental de la création est précisément celui que va reprendre et approfondir le théologien samoan Tofaeono, en y adjoignant, comme nous allons le découvrir, celui de la famille élargie.

\section{Le concept d'«Aiga » chez Ama Amalele Tofaeono}

Ama'amalele Tofaeono Siolo est originaire des Samoa Occidentales. Pasteur de l'Église Congrégationaliste Chrétienne, il étudie la théologie au Collège Théologique de Kanano Fou situé aux Samoa Américaines, de 1986 à 1990. Issu d'une famille modeste, il travaille temporairement pour les Parcs et Réserves Nationaux. De son propre aveu, il ne comprend pas vraiment le sens de ce « job»: " pourquoi se mettre au service des arbres, des oiseaux, des poissons et l'environnement plutôt qu'au service des hommes ? " Pourtant, cette expérience aura un impact sur sa recherche théologique : " mais en même temps, cela m'a confronté à la réalité de mon lien et de ma connexion au fanua ${ }^{9}$ " (Tofaeono, 1993 : xii).

De 1991 à 1993, il poursuit ses études au PTC à Fidji pour une licence en théologie. Il retourne ensuite à Kanano Fou où il enseigne une année avant de partir en Allemagne où il soutient son doctorat en théologie en 2000.

De retour dans le Pacifique, il enseigne la dogmatique et l'éthique au PTC jusqu'en 2004. Il est sollicité par des universités nord-américaines pour de courtes périodes d'enseignement, notamment à la Vancouver School of Theology (University of British Columbia). Il obtient un prix de l'Université de Berkeley en $2002{ }^{10}$ pour une recherche consacrée à " l'écothéologie du Moana (Océan) » et un autre décerné par l'Union Seminary de New York pour un cours consacré à la «christologie du Pacifique ». En 2006, Tofaeono s'installe à Auckland où il dessert une paroisse d'immigrés samoans.

Le point de départ de la théologie de Tofaeono n'a rien de proprement religieux mais réside dans la prise au sérieux des menaces écologiques qui pèsent non seulement sur son pays mais plus généralement sur l'ensemble du Pacifique.

8. La sotériologie est la doctrine du Salut.

9. Fanua est l'équivalent samoan du fidjien vanua ou du tahitien fenua: la terre et la mer, le pays et tout ce qu'il contient.

10. CNTS Science et Religion Course Award Competition. 
Ce n'est que dans un deuxième temps qu'il aura recours aux catégories " religioculturelles » samoanes.

Les prolégomènes du raisonnement théologique de Tofaeono reposent sur trois facteurs : la crise écologique mondiale et en particulier la pollution maritime; le dévoiement du christianisme par la culture occidentale sous l'ère missionnaire, mais également depuis l'antiquité et l'influence hellénistique ; la culture samoane à redécouvrir comme "clé herméneutique » de tout système théologique.

Comment retrouver un christianisme authentique, c'est-à-dire fidèle à la fois à son inspiration scripturaire et à la faa Samoa, la culture et manière de vivre samoane ? La solution proposée par Tofaeono est d'élaborer une écothéologie. Au centre d'une telle théologie, la création et la rédemption ont une place essentielle. La nécessité de sortir d'une vision anthropocentrique de la création est mise en avant : des échos et des traces du divin se manifestent dans l'héritage religio-culturel relaté dans les expressions mythiques et les traditions orales de Samoa (Tofaeono, $2000: 20$ ).

Au centre de la faa Samoa se trouvent la maison et la cellule familiale - aiga concept capital pour la pensée écothéologique de Tofaeono. Aiga exprime en effet à lui seul la plénitude de la vie samoane. Il relie le divin et l'ordinaire dans une synthèse existentiale. Le concept d'aiga dans son acception écologique comporte également une orientation existentielle : bien que lié à un lieu précis et situé dans le temps, il élargit son horizon aux sources de la vie - Dieu, esprits et ancêtres - et anticipe simultanément le futur de la vie. C'est une institution et un concept qui donnent forme à la vie de la communauté dans toutes ses dimensions. Pour Tofaeono, aiga est le paradigme à partir duquel tout Samoan serait à même de saisir le concept chrétien de Trinité dans toute sa profondeur : "Je suggère l'image indigène de l'aiga ${ }^{11}$ comme métaphore théologique unifiante » (Tofaeono, 1993 : 95).

Dans un second temps, Tofaeono articule l'aiga au modèle théologique de La Trinité. En examinant plus particulièrement sa christologie, il apparaît que, comme chez Tuwere, les concepts de mana et de tapu paraissent les plus adéquats pour qualifier le Christ, tout en y ajoutant sa place bien particulière au sein l'aiga puisqu'il est serviteur, tautua. Ce mot exprime une relation interactive de tous les membres de l'aiga avec les puissances ou agents cosmiques mana et tapu. Le Christ est compris comme une énergie surnaturelle et sainte qui fait le pont entre l'ordinaire et le spirituel. Or en tant que Sagesse, Christ est « énergie transcendante » et "médiateur de la vie ».

De plus, le Christ serviteur (tautua) renforce l'alliance du cosmologique et de l'anthropologique : "dans le contexte de l'aiga, tautua est accompli pour le bien commun et la sauvegarde de tous les membres de la famille. » Jésus et les

11. Home : famille ou maisonnée. 
pauvres, tel que Luc nous le présente, trouve un écho pertinent dans la situation des Samoans : "Comme gens de la terre, les Samoans et les Océaniens ne sont pas vraiment concernés par le problème de la pauvreté et dans un certain sens, personne ne meurt de faim ou n'est sans abri. Mais les forces du développement moderne technologique et économique sont maintenant une menace. Ces forces introduisent un esprit de compétition qui entraîne l'individualisme. Ces forces essaient d'introduire la privatisation et la commercialisation du système foncier, exacerbent l'industrie de la pêche et tentent d'élever tout le monde aux standards des autres» (Tofaeono, 2000 : 256-257). Recourant à l'étymologie, Tofaeono explique que dans la conception samoane du chef, " celui-ci est œil (mata) posé sur l'ai (prospérité) dont dépend l'aiga ». Ainsi, par le biais de ce titre de matai, la christologie établit un pont entre écologie et économie, conformément à la double connotation de l'association aigaloikos.

\section{Continuités et mutations du discours théologique océanien contemporain}

Ces trois théologiens océaniens entendent s'inscrire dans la continuité du discours dogmatique protestant traditionnel. À cet égard, leur passage obligé par le dogme de la Trinité est symptomatique, même si ce dernier se voit revisité dans son contenu dogmatique au prix d'un effort herméneutique considérable. Havea en particulier, ne remet jamais en cause ni le dogme trinitaire, ni la place centrale de la figure du Christ dans l'économie du salut. Son combat pour une contextualisation de l'Évangile porte selon lui sur un changement de méthode et non sur un contenu dogmatique qui entend rester très classique et fidèle à l'héritage missionnaire anglais du XIX ${ }^{\mathrm{e}}$ siècle.

\section{La « disponibilité » de l'Évangile}

Cet effort ne masque cependant pas une première mutation : la réinterprétation de la tradition est réalisée à travers ce qu'il appelle la disponibilité de l'Évangile dès la Pentecôte. Selon lui, les effets de l'ephapax christique ne se sont pas limités aux frontières connues par les auteurs bibliques, mais ont touché tous les rivages de la terre y compris les peuples du Pacifique. De sorte que l'action d'évangélisation des missionnaires ne se présente plus comme une "première annonce " mais comme une "révélation " d'une grâce déjà là, " disponible ». Cette thèse suscitera - et suscite encore - quelques résistances ${ }^{12}$.

12. Bruno Saura (Saura, 2003) a relevé ce point et montré la contiguïté de pensée entre Havea et la " nouvelle théologie " tahitienne qu'il étudie à travers l'œuvre du théologien ma'ohi Turo Raapoto : « Havea fait à la fois preuve de plus de prudence [que Raapoto], dans la mesure où il admet que cette présence n'était qu'inconsciente, mais aussi plus d'audace lorsqu'il signifie que les scènes de la Pentecôte ou le sacrifice du Christ ont eu un effet à l'échelle de la terre entière, y compris des îles du Pacifique ". 
L'herméneutique du symbole entraîne donc inévitablement une réinterprétation - voire une subversion - du dogme traditionnel dans la mesure où ce dernier ne sert plus que de support ou de caution à l'élaboration d'une nouvelle démarche fondée sur le donné anthropologique. C'est précisément ce processus que la missiologie qualifie de contextualisation. La primauté chronologique de la culture sur l'Évangile n'implique nullement un développement parallèle des deux pôles mais aboutit à la création d'un nouveau cadre de référence, un « troisième espace ni purement occidental, ni purement indigène » comme le définit J. Barker et qui est tout sauf immuable.

\section{Une contextualisation critique}

Dans cette perspective, l'ouvrage majeur de Tuwere, Vanua, Towards a fijian theology of place constitue une seconde mutation en tant qu'illustration de la contextualisation de l'Évangile à partir de la question de la terre et de l'identité. La nouveauté de Tuwere pour la théologie océanienne réside avant tout dans ses efforts de systématisation de la problématique de la contextualisation qu'il est légitime de qualifier de critique : "La vraie contextualisation, c'est donner à l'Évangile la primauté, l'autorité et le pouvoir de pénétrer chaque culture pour que se fasse entendre à l'intérieur de celle-ci la Parole qui est à la fois oui et non, haine et amour, jugement et grâce » (Tuwere, 2002 : 11). Mais l'appartenance d'une communauté chrétienne à l'oikuménè est pour Tuwere tout aussi importante en ce qu'elle peut prévenir des tentations et dérives culturalistes et communautaristes : "Notre tendance naturelle à l'égoïsme nécessite la correction de ceux qui partagent la vie en Christ mais qui habitent différentes situations culturelles. La théologie contextuelle doit accepter des corrections de la part de ceux qui partagent la même foi que nous mais qui appartiennent à une autre culture » (Tuwere, 2002 : 12). L'Évangile fonctionne ainsi pour Tuwere comme un correctif à la culture en raison de ses implications éthiques. Dès lors, les mutations culturelles ne puisent pas leur source dans la tradition mais bien dans l'herméneutique, dans l'effort d'interprétation du texte qui est la marque de fabrique du christianisme, en particulier du protestantisme.

\section{Un christianisme holiste}

Ce criticisme contraste avec le projet théologique de Tofaeono, porteur d'une troisième mutation par l'articulation entre concepts religio-culturels samoans et questions écologiques. L'Évangile est ici conçu d'une manière résolument inclusive, "w/holistic» selon ses propres termes, c'est-à-dire à la fois holiste et porteuse de plénitude. Penser à partir de ses propres concepts culturels - et non plus selon un modèle importé - tel est le défi relevé par le théologien samoan. En mettant l'accent sur l'aspect interrelationnel de toutes les créatures comme ancrage - voire image - de la relation trinitaire, il s'inscrit résolument dans une 
démarche théologique relevant de l'orthopraxie, se détachant nettement de paradigmes théologiques plutôt basés jusque-là sur un raisonnement dichotomique. Se détournant d'un point de vue qu'il juge trop " anthropocentrique ", il entreprend une théologie "théocentrique et/ou théo-écologique " dont la principale implication sera de déboucher sur une "spiritualité éco-éthique " témoignant de l'activité du Dieu vivant au sein de la création (Tofaeono, 2000 : 289).

La mise en perspective de ces trois théologiens permet de mesurer le déplacement du discours théologique par rapport à la culture. Tous revendiquent une continuité quasi apostolique de l'oikuménè mais chacun développe un éthos bien particulier : foncièrement culturel pour Havea, politique pour Tuwere, écologique pour Tofaeono. Cette distorsion entre une continuité formelle affichée sur le plan de la foi, œcuménique, et la réalité d'une nouvelle foi, en phase avec la culture, relève selon nous de la double problématique du syncrétisme et du traditionalisme, dont l'une des caractéristiques serait, selon A. Babadzan, la "volonté délibérée de réinventer le passé en fonction des intérêts du présent " (Babadzan, 2009 : 207).

\section{Essai de périodisation de la théologie contemporaine}

$\mathrm{Au}$ terme de la présentation de ces trois théologiens du Pacifique, nous voudrions proposer une périodisation historique de la contextualisation de ce discours religieux spécifique depuis les années 1960. Nous croyons pouvoir y discerner trois phases.

\section{Des années 1970 aux années 1980 : une théologie de l'identité}

Dans cette période émergent une théologie et une identité océaniennes grâce à Sione Amanaki Havea. Il introduit l'utilisation de symboles culturels - dont la célèbre " théologie du coco ", la nécessaire révision d'une définition de la Trinité et de la christologie avec la recherche d'un «Christ du Pacifique » et le passage d'une théologie occidentale dialectique et critique à une théologie océanienne du don et de la célébration. En 1983, S. A. Havea conduisit la délégation des Églises du Pacifique à l'Assemblée du COE de Vancouver. Cette manifestation marque à la fois le sommet et la fin de son influence directe sur la pensée théologique et sur la vie des Églises au niveau régional. C'est à cette occasion que le monde œcuménique reconnut pour la première fois le continent océanien comme une entité propre - détachée de l'association habituelle "Asie-Pacifique ». L'assemblée put prendre toute la mesure de ce que C. Forman (1986) appelle "l'identité chrétienne du Pacifique ». En cherchant la voie d'une " pacifitude de la théologie ", Havea ne fait au fond que reproduire, sur un mode religieux, le discours politique 
et culturel nationaliste de la Pacific Way ${ }^{13}$ sous-tendu par l'idéologie de la coutume qui apparaît à la fin des années 1960 au moment où la plupart des États insulaires du Pacifique accèdent à leur indépendance ${ }^{14}$. Cette identité océanienne, ce Pacific way dérive aussi bien du passé, des intérêts communs et des attitudes culturelles traditionnelles, que du présent et du futur en raison des défis et dangers auxquels les chrétiens insulaires du Pacifique sont déjà ou vont être incessamment confrontés. La théologie contextuelle de Havea trouve ici sa limite: face à une mondialisation qui ne dit pas encore son nom, ne risque-t-elle pas d'articuler trop artificiellement des symboles culturels spécifiques océaniens à une réalité marquée par l'urbanisation et son corollaire, l'exode rural, des échanges économiques croissants et le développement rapide des communications, vecteurs de nouvelles visions du monde et donc de compétition entre Églises, signe distinctif de la "troisième vague » définie par J. Barker ${ }^{15}$ ?

Outre cet aspect sociopolitique, le désir de Havea de «théologiser » sur des bases proprement océaniennes, et non plus dans un cadre occidental prédéfini, est également à replacer au sein des débats théologiques du mouvement missionnaire et œcuménique mondial. Présent dans les instances œcuméniques mondiales de 1954 à 1983, le théologien tongien en reprend les grandes orientations. Cela apparaît de manière flagrante lorsqu'on met en regard ses idées et les trois principaux thèmes de la conférence du Conseil International des Missions (CIM) tenue à Wilingen en 1952 : la réaffirmation trinitaire de la mission, avec le fameux mot d'ordre «missio Dei »; l'importance de la figure du Christ incarné et solidaire des hommes de son temps, la définition de l'Église comme " peuple de Dieu » dans le monde (Zorn, 1998). Cette parenté se vérifie également par rapport au refus d'une régionalisation de l'Évangile et son pendant : l'affirmation de l'unité et de l'universalité de l'Église. Selon Marc Spindler (Spindler, 1967 : 71), c'est à Willingen que la «théologie de l'enracinement de l'Évangile dans la culture trouve sa propre limite : il ne s'agit pas d'être enraciné dans le sol mais d'être en relation avec le sol. L’Église ne peut être enracinée qu'en Christ. » De même, il faut relever que la métaphore utilisée par Havea « de la plante et du pot » exprimant la transplantation artificielle de l'Évangile dans son emballage occidental est un emprunt direct à Daniel T. Niles : "nous voulons la plante de

13. D’après Frédéric Rognon, on peut synthétiser la Pacific Way comme un discours mettant en relief les valeurs suivantes : "sens de l'hospitalité, fraternité et unité, recherche du consensus, soin (care) pour le prochain, sens de la vie communautaire et de la solidarité intergénérationnelle, respect des anciens et des chefs, etc. » (Rognon, 1991: 200).

14. Pour mémoire, au cours de la décennie 1970-1980 les indépendances des pays insulaires du Pacifique se sont échelonnées comme suit : Samoa Occidentales en 1962, Nauru en 1968, Fidji et Tonga en 1970, Papouasie Nouvelle-Guinée en 1975, Tuvalu (Ellice) en 1978, Kiribati (Gilbert) en 1979, Vanuatu (Nouvelles-Hébrides) en 1980 (De Dekker, 1985).

15. Selon J. Barker, la «troisième vague » missionnaire s’étendant du milieu des années 1960 à nos jours est caractérisée par la décolonisation des missions, la naissance d'Églises nationales et l'indigénisation du clergé. Il conviendrait d'ajouter la régionalisation des institutions qui font émerger la conscience d'une spécificité océanienne chrétienne face aux autres continents. 
l'Évangile et non le pot et la terre dans lesquels vous [i.e. les missionnaires] nous l'apportez " (Spindler, 1967 : 51). Cette citation - reprise textuellement par Havea - est typique du reproche fait à la Mission par les jeunes Églises au lendemain de leur autonomie que M. Spindler qualifie "d'occidentalisme». Enfin, la recherche par S. A. Havea d'une nouvelle herméneutique théologique s'affirmant contre le "moule » théologique occidental se situe dans le droit fil de la conférence missionnaire mondiale de Bangkok ${ }^{16}$ en 1972, où « la revendication d'une théologie contextuelle apparaît sur la scène internationale, comme contestation de la domination missionnaire » (Matthey, $1998: 51$ ).

Replacée dans le contexte missiologique international de l'époque, la pensée de Havea correspond donc parfaitement aux aspirations du temps. Relevons toutefois que les thématiques qui s'en dégagent s'orientent différemment par rapport aux autres continents. En effet, elles ne prennent pas un tour politique comme dans le cas de la théologie de la libération latino-américaine par exemple. Elles semblent davantage s'orienter vers la quête d'identité que connaît la théologie africaine à la même époque mais, contrairement à cette dernière, elles ne débouchent pas vers une " théologie du cri ", de la souffrance du colonisé obligé de se reconstruire dans tous les domaines de sa vie ${ }^{17}$. Elles se démarquent encore de la théologie asiatique davantage préoccupée par le pluralisme des religions. On retiendra cependant ici une proximité entre l'Asie et le Pacifique dans le recours au symbole et à la diversification des expressions théologiques non seulement à travers le discours mais aussi par la danse et la ritualité ${ }^{18}$.

\section{Des années 1980 à la fin des années 1990 : le temps de l'affirmation de la culture océanienne et de la contextualisation de la théologie}

Cette époque est marquée par Sevati Tuwere qui promeut inlassablement le concept d'une théologie spécifiquement océanienne. Il se démarque toutefois de son prédécesseur en systématisant davantage sa réflexion, notamment en plaçant la contextualisation au centre de sa démarche théologique. Deux influences majeures s'exercent selon nous sur sa théologie de la terre : d'une part celle de Moltmann, à travers la primauté de la création sur la rédemption dans l'économie du salut, ainsi que par l'utilisation du modèle trinitaire définissant les relations entre les personnes de la Trinité, "communauté en communion », comme

16. Le moratoire en question, demandé par les théologiens "du Sud ", stipulait le retrait de tout missionnaire expatrié, afin de laisser aux Églises locales le soin de se forger leur propre programme et d'assurer leur autosuffisance (Matthey, $1998: 51-52$ ).

17. Sur ce point, voir K. Blaser qui discerne une évolution de la théologie africaine allant de l'inculturation à la reconstruction (Blaser, 1995 : 278-280).

18. Blaser donne l'exemple de la prestation de Mme Chung lors de l'assemblée mondiale du COE à Canberra en 1991 et conclut : « un nouveau mode de réflexion théologique et missionnaire s'affirme ici : on est dans le contexte du dialogue entre les cultures et il s'agit de se communiquer les symboles et les rituels, de se communiquer par eux » (Blaser, 1995 : 289). 
matrice possible des relations humaines; d'autre part celle la réflexion initiée par le COE centrée sur la thématique "Justice, Paix et sauvegarde de la création » plaçant au cœur du débat théologique la question de la justice sociale et un début de réflexion écologique. Ces deux courants repérables dans les écrits de Tuwere, ont permis à la théologie du Pacifique et aux Églises de ne pas rester dans la problématique de l'identité culturelle, mais de s'ouvrir davantage au monde. Nous qualifions cette ère nouvelle, la décennie 1980-1990, de «temps de l'affirmation " de la théologie contextuelle du Pacifique, par opposition au temps précédent où il s'agissait de construire et de rendre visible une entité œcuménique commune.

Au niveau ecclésial régional on observe, sinon une influence directe, du moins une forte convergence entre la ligne théologico-éthique de théologiens professionnels tels que Havea et Tuwere d'une part, et la ligne sociopolitique et économique de chrétiens laïcs engagés pour la justice sociale tels que Suliana Siwatibau ${ }^{19}$. Régulièrement invitée comme personne-ressource dans les assemblées d'Églises, ses discours relèvent de la même veine anthropothéologique que les écrits de Tuwere ${ }^{20}$.

De plus, il est également un autre lieu où l'impact théologique de Tuwere est très prononcé : l'Association des Écoles de Théologie du Pacifique Sud (SPATS). Il participe activement à la vie et au renouveau de l'association qui était un peu en sommeil depuis les années 1970. De 1985 à 1999, il n’intervient pas moins de sept fois en tant qu'organisateur et formateur de réunions théologiques régionales. Si l'on excepte sa période de formation en Australie de 1989 à 1992, on s'aperçoit que Tuwere a nourri la pensée théologique dans le Pacifique insulaire pendant une bonne quinzaine d'années. À cela s'ajoute son engagement politique personnel en 2000 apportant un surplus de crédibilité éthique à sa théologie propre à renforcer son audience.

\section{Des années 1990 au début des années 2000 : le temps du dialogue}

Enfin, dans cette dernière décennie du $\mathrm{XX}^{\mathrm{e}}$ siècle s'ouvre le temps du dialogue entre la culture océanienne et l'oikuménè avec Ama Tofaeono. Sa thèse sur l'aiga comme paradigme d'une écothéologie samoane extensible aux autres cultures du Pacifique, et ses recherches sur l'océan comme lieu de la révélation dans la création et la culture témoignent d'une étape supplémentaire dans la contextualisation de la théologie. Au niveau régional elle trouve un écho dans la recherche

19. S. Siwatibau est intervenue à chaque assemblée de la PCC en 1981, 1986 et 1991. Elle a travaillé par la suite pour le compte du gouvernement fidjien, ainsi que pour des ONG, surtout dans le cadre de programmes de l'ONU.

20. «Nous n'avons pas su reconnaître que les esprits que nos ancêtres adoraient, les gardiens de notre environnement, n'étaient que des manifestations de notre conception encore limitée du seul Dieu véritable que nous adorons maintenant en tant que chrétiens. [...] Dieu, le Créateur, le Tout-Puissant, celui qui sait tout, est partout, il n'a pas changé : c'est notre conception de ce même être qui a changé avec la christianisation (Siwatibau, 1991:19). 
menée à Tahiti sur l'identité ma'ohi et le fenua comme lieu de la révélation de l'identité de l'homme et de la théophanie. Cependant, elle s'en démarque sensiblement en ce qu'elle entre en dialogue critique avec des traditions chrétiennes anciennes ou culturellement éloignées, de même qu'elle se confronte aux théologies occidentales du Vieux et du Nouveau continent.

Comme ses prédécesseurs Havea et Tuwere, Tofaeono est grandement influencé par les orientations théologiques du COE, en particulier par la réflexion des années 1990 autour de "Justice, Paix et Sauvegarde de la création ». Lui aussi reprend les thèmes moltmanniens de la création et de La Trinité comme modèle de relations justes, tout en insistant, davantage que Tuwere, sur la christologie. Le "Christ cosmique " ainsi que le "Christ de l'Esprit » cher au théologien allemand (Moltmann, 1993) lui fournit le cadre théorique permettant d'introduire les notions culturelles samoanes du Christ comme chef dans sa double fonction de souverain (matai) et de serviteur (tautua), attentif à la situation des plus pauvres : "Jésus prêche un Évangile réaliste et non idéaliste, assurant aux pauvres une nouvelle réalité vivante»(Tofaeono, $2000: 258$ ).

À côté de ce maître, la réflexion plus politique de Leonardo Boff, Paulo Freire et Dorothee Söllese se retrouve également sous la plume de Tofaeono, ainsi que celle du théologien du Nicaragua au service du COE, Julio de Santa Ana, sur les questions de la justice économique et de la colonisation. Enfin, l'auteur se dit lui-même impressionné par ses rencontres avec un théologien amérindien, George Tinka, et surtout avec la théologienne américaine Sally Mac Fague, à la fois pour sa critique de l'image paternaliste de Dieu et pour l'orientation écologique de sa théologie ${ }^{21}$.

Par rapport à Havea et Tuwere, nous voudrions souligner la diversité de ces théologiens composant l'univers de pensée de Tofaeono à côté des ressources propres de sa culture. Certes, tous se retrouvent autour de préoccupations écothéologiques, mais ce qui retient avant tout notre attention est la pluralité géographique et culturelle de ces liens, puisque le "vieux " continent européen où l'auteur a rédigé l'essentiel de sa thèse est aussi bien représenté que le « nouveau » continent américain. Nous voyons là l'illustration du projet de l'auteur : loin de s'enfermer dans une théologie contextuelle identitaire et exclusiviste, il s'attache plutôt à faire dialoguer des théologies de tous horizons pour contribuer à un enrichissement mondial de la théologie dans le cadre de l'oikuménè. En d'autres termes, chaque tradition peut, selon lui, apporter sa pierre à l'édifice mondial et œcuménique que représente le projet d'une éco-théologie, quand bien même celleci s'incarnerait dans une culture particulière, celle des Samoa en l'occurrence.

Puisant à ces différentes sources, la contextualisation de la théologie océanienne se veut à présent consciente de son déploiement dans le monde œcuménique au niveau mondial, non seulement pour y apporter sa contribution mais

21. Entretien avec l'auteur, 16 septembre 2006, Suva. 
aussi pour s'en enrichir. Elle devient une recherche exigeante, académique, inclinant parfois au repli identitaire, tentant d'assumer ses multiples héritages historiques, bibliques et culturels dans des synthèses où discours religieux et culturels s'auto-légitiment sans cesse.

En examinant le processus de contextualisation de la théologie dans le Pacifique d'un point de vue missiologique, on constate que "l'élite » de la pensée chrétienne protestante post-missionnaire a sans cesse cherché à dépasser la problématique de la continuité culturelle et des mutations sociales. S'appuyant sur une herméneutique du symbole, elle s'efforce de faire évoluer le cadre culturel dans un sens éthique, voire éthico-écologique. On peut toutefois s'interroger sur la portée ecclésiale de ce discours intellectuel idéalisé sur la terre, vanua, ou la maisonnée, aiga sur le terrain, dans la mesure où il est produit par des théologiens précisément de plus en plus détachés de ces réalités. Dans les sociétés océaniennes, la fonction du théologien se situe à la confluence de l'homme de pouvoir, a fortiori s'il est investi par une institution comme cela a été le cas pour Havea et Tuwere, et de la figure de l'intellectuel, du sage, dépositaire de la sacralité d'une parole transcendante au poids non négligeable dans le champ social ${ }^{22}$.

\section{Conclusion}

Au terme de la présentation de ces trois théologiens océaniens contemporains, une question demeure toutefois : quelle est la portée effective - au-delà du seul discours - sur les institutions ecclésiales et la vie paroissiale " de base » de cette parole théologique se prétendant spécifiquement océanienne, reprenant sur un mode religieux les propos politiques sur la coutume fondés sur l'idéologie de la Pacific Way, exaltant le partage de la terre dans une paix fraternelle ou privilégiant une approche cosmologique dépassant le seul espace océanien ? Étudiant un phénomène contemporain dont les effets se poursuivent toujours sous nos yeux, il serait présomptueux d'apporter ici une réponse définitive. Deux caractéristiques méritent cependant d'être soulignées.

La première consiste dans la localisation de la prise de parole de ces théologiens et de leur distanciation. En effet, tous trois ont enseigné, écrit et développé leurs thèses à partir de Suva, capitale régionale géopolitique, académique, mais aussi ecclésiale, puisqu'elle abrite à la fois la PCC et les deux collèges de formation théologique supérieurs. Cette position centrale a très certainement favorisé la diffusion et la médiatisation - ceci se vérifie en particulier pour Tuwere - de leur théologie, à l'intérieur de la zone Pacifique, mais aussi en direction de l'extérieur, dans les milieux académiques et ecclésiaux. En revanche,

22. F. Douaire-Marsaudon mentionne par exemple la prise de position publique de Havea pour une séparation entre l'Église et l'État, alors qu'il était président de la Free Wesleyan Church of Tonga, une Église créée par la monarchie tongienne au XIX ${ }^{\mathrm{e}}$ siècle et dépendant étroitement de son pouvoir (Douaire-Marsaudon, 2010 : 75). 
excepté pour Tuwere, l'impact de leur théologie dans leur île d'origine est plus mitigé : l'un des opposants les plus farouches à la contextualisation de la théologie à partir du symbolisme culturel telle que le défendait Havea est l'un de ses compatriotes, Ma'afu Palu, enseignant au Collège théologique de Sia'atoutai ${ }^{23}$. Quant à Tofaeono, il ne semble pas que son enseignement écothéologique trouve à ce jour un écho significatif dans les collèges théologiques des Samoa de Malua, Piula ou Kanana Fou. En outre les obstacles « administratifs 24 » l'ayant empêché de reprendre son enseignement au PTC peuvent laisser penser que ses thèses sont loin de faire l'unanimité au sein de toutes les Églises du Pacifique, seules responsables de la nomination des enseignants de cette institution. Il semble donc que l'impact de ce discours religieux culturel se soit porté sur un cercle restreint : les étudiants du PTC et une intelligentsia océanienne de théologiens participant régulièrement aux colloques théologiques organisés sous l'égide de la SPATS ou lectrice de son principal outil de diffusion, le Pacific Journal of Theology.

La deuxième caractéristique qui tend à infléchir l'impact de ce nouveau discours religieux tient à la propension des théologiens océaniens, dont Tuwere et Tofaeono, à s'exiler pour enseigner en Australie ou en Nouvelle-Zélande ${ }^{25}$. Outre de meilleures conditions matérielles, ce positionnement " extérieur » présente le double avantage d'une plus grande liberté de parole par rapport à leur Église d'origine, ainsi que d'un moindre isolement, donc d'une meilleure diffusion de leurs idées au niveau international, comme en témoigne par exemple l'attribution académique de deux prix théologiques pour Tofaeono, laissant à penser que ce dernier est plus connu aux États-Unis qu'à Samoa. Dans le même ordre d'idée, nous avons montré ailleurs que la "théologie de la célébration » de Havea ne s'est pas vraiment traduite sur le plan liturgique au niveau des congrégations mais plutôt dans le cadre restreint de célébrations « exceptionnelles » régionales (Vidal, $2011: 336)$.

Finalement, le plus grand défi de la contextualisation de la théologie en Océanie sera peut-être, dans les années qui viennent, de parvenir à saisir la nature constamment dynamique des contextes sociaux et des mutations religieuses à l'œuvre dans la région, largement conditionnés par le double mouvement de l'urbanisation et de la mondialisation.

Gilles VIDAL

Centre Maurice-Leenhardt de recherche en Missiologie Institut Protestant de Théologie - Faculté de Montpellier gilles-louis.vidal@univ-montp3.fr

23. Il s'agit de Ma'afu 'o Tu'itonga Palu. Voir Palu, 2002.

24. Selon les termes mêmes de Tofaeono lors d'un entretien personnel.

25. Plusieurs enseignants-chercheurs ont choisi cette voie de l'exil après un temps d'enseignement au PTC: Toa Finau, John Havea, Jovili Meo, etc. 


\section{Bibliographie}

BABADZAN Alain, 2009, Le spectacle de la culture. Globalisation et traditionalismes en Océanie, Paris, L'Harmattan.

BLASER Klauspeter, 1995, La théologie au Xx ${ }^{e}$ siècle, Lausanne, L'Âge d'Homme.

De Dekker Paul, 1985, "Indépendances et dépendances dans le Pacifique insulaire ", Vingtième Siècle, 6-6, p. 23-38.

Douarre-Marsaudon Françoise, 2010, « Mémoire trouble. Histoire d'une recomposition politico-religieuse en Polynésie (Tonga) ", in Douaire-Marsaudon Françoise \& Weichart Gabriele (éds.), Les dynamiques religieuses dans le Pacifique. Religious Dynamics in the Pacific, Les cahiers du CREDO, Pacific Credo Publications, p. 57-83.

ERnst Manfred (éd.), 2006, Globalization and the Re-shaping of Christianity in the Pacific Islands, Suva, PTC.

FIELD Michaël et alii, 2005, Speight of Violence: Inside Fiji's 2000 Coup, Auckland, Reed. Forman Charles, 1986, The Voice of Many Waters, Suva, Lotu Pasifika Publications.

Havea Sione Amanaki, 1977, The Pacifiqueness of Theology, An Address delivered at the Theological Club, Suva, 11 october 1977, [s.l.], [s.n.], [s.d.].

Havea Sione Amanaki, 1987, "Christianity in the Pacific Context ", in Havea S. A. (éd.), South Pacific Theology: Papers from the Consultation on Pacific Theology, Papua New Guinea, January 1986, Oxford/Paramatta, Regnum Books / World Vision International South Pacific.

MatTHEy Jacques, 1998, «Les conférences universelles des missions de 1947 à 1996, II. Jalons de la pensée missionnaire protestante œcuménique des années 1970 aux années 1990 », Perspectives Missionnaires, 36-2, p. 51-52.

Moltmann Jürgen, 1993, Jésus, le messie de Dieu, [éd. allemande : 1989], trad. Joseph Hoffmann, Paris, Cerf, coll. Cogito Fidei.

Palu Ma'afu 'o Tu'itonga, 2002, « Pacific Theology », Pacific Journal of Theology, Series II, 28, p. 21-53.

RognON Frédéric, 1991, Conversion, syncrétisme et nationalisme. Analyse du changement religieux chez les Mélanésiens de Nouvelle-Calédonie, Thèse de doctorat en ethnologie, Université de Paris X-Nanterre.

SAURA Bruno, 2003, "Naissance d'une pensée millénariste ethnique et développement du nationalisme à Tahiti », Bulletin de la Société des Études Océaniennes, 295, p. 37-38.

SiWATibau Suliana, 1991, "Renaître pour une espérance vivante, proclamer une espérance vivante ", Conférence des Églises du Pacifique, Rapport de la Sixième Assemblée, trad. Anne Quehen, Suva, Lotu Pasifika Productions.

SPINDLER Marc, 1967, La mission, combat pour le Salut du monde, Neuchâtel, Delachaux et Niestlé.

Tofaeono Ama'amalele, 1993, A Quest for a Samoan Theology of Creation, Thesis B.D., Suva, PTC.

Tofaeono Ama'amalele, 2000, Eco-Theology: AIGA the Household of Life, a perspective from living myths and traditions of Samoa, Erlangen, Erlanger Verlag für Mission und Ökumene. 
Tuwere Ilaitia Sevati, 1989, "Justice and Peace in the Womb of the Pacific ", Pacific Journal of Theology, Series II, 1, p. 8-16.

Tuwere Ilaitia Sevati, 1992, "Emerging Themes for a Pacific Theology », Pacific Journal of Theology, Series II, 7, p. 49-55.

Tuwere Ilaitia Sevati, 1994, "A Theological Response to "Winds of Change" ", Pacific Journal of Theology, Series II, 12, p. 27-31.

Tuwere Ilaitia Sevati, 1995, "An Agenda for the Theological task of the church in Oceania ", Pacific Journal of Theology, Series II, 13, p. 5-20.

Tuwere Ilaitia Sevati, 1997, "Response to Dr Dass ", Pacific Journal of Theology, Series II, 17, p. 84-91.

Tuwere Ilaitia Sevati, 2002, "What is Contextual Theology: a view from Oceania ", Pacific Journal of Theology, Series II, 27, p. 7-20.

VIDAL Gilles, 2011, Les théologies contextuelles dans le Pacifique Sud au XXe siècle. Analyse des conditions de production d'un discours religieux en situation, thèse de doctorat en histoire contemporaine et en théologie, Université Paul Valéry Montpellier III/Institut Protestant de Théologie - Faculté de Montpellier.

ZoRn Jean-François, 1997, «La contextualisation : un concept théologique ? ", Revue d'Histoire et de Philosophie Religieuse, 77-2, p. 171-189.

ZoRn Jean-François, 1998, "Les mutations du monde missionnaire et œcuménique de 1947 à 1963 ", Perspectives Missionnaires, 36-2, p. 39-41.

ZoRN Jean-François, 2004, La missiologie, émergence d'une discipline théologique, Genève, Labor et Fides.

\section{La contextualisation de la théologie protestante comme lieu de changement du christianisme en Océanie}

Cet article propose de considérer le discours religieux chrétien océanien contemporain, dans sa version protestante, comme lieu privilégié de changement du champ social. Selon une approche dialogique entre théologie, anthropologie et histoire, trois concepts-clés élaborés par trois "figures de proue " de la théologie protestante océanienne contemporaine sont tout d'abord étudiés : la communauté chez le Tongien Sione Amanaki Havea, le vanua (le pays) chez le Fidjien Sevati Tuwere et l'aiga (la famille élargie) chez le Samoan Ama Amalele Tofaeono. Dans un deuxième temps, il s'agit de montrer comment ces trois concepts s'inscrivent, dans le discours théologique, soit dans une certaine continuité par rapport à la théologie missionnaire héritée de la fin $d u X_{X}{ }^{e}$ et du début de XX ${ }^{e}$, soit au contraire dans une transformation entre contextualisation critique et christianisme de type holiste. Puis une périodisation de la théologie protestante océanienne des années 1970 aux années 2000 est proposée, partant d'une "théologie de l'identité " se voulant ancrée dans la tradition pour aboutir à une théologie interculturelle fondée sur le dialogue oecuménique. La conclusion tente enfin d'évaluer la portée effective sur les institutions ecclésiales de ce discours tenu, comme on le découvrira, par des intellectuels urbains largement influencés par l'idéologie panocéanienne de la Pacific Way.

Mots-clés : christianisme, Océanie, théologie contextuelle, missiologie. 


\section{The contextualisation of protestant theology in Oceania as a Sign of a Changing Christianity}

This paper sets out to consider protestant religious discourse in contemporary oceania as a particularly valuable place to observe change in society. In the first part, three key concepts developed by three "figureheads" of contemporary protestant theology in the Pacific are discussed: the concept of community in the work of the Tongan Sione Amanaki Havea, vanua (land) in that of the Fijian theologian Sevati Tuwere, and aiga (or extended family) in that of the Samoan Ama Amalele Tofaeono. The second part of the paper shows how these three concepts have remained in theological thinking either as a continuation of missionary theology from the end of the $19^{\text {th }}$ century and beginning of the $20^{\text {th }}$ century or, on the other hand, as a transformation between critical contextualisation and a holistic type of Christianity. Next, a division of the protestant theology in the Pacific from the 1970s to the 2000s in different phases is given, showing the evolution from a theology of identity based on tradition to an intercultural theology based on ecumenical dialogic. Finally, the conclusion evaluates the effective impact on church institutions of the discourse of urban intellectuals broadly influenced by the Panoceanian "Pacific way" ideology.

Key words: Christianity, Oceania, contextual theology, missiology.

\section{La contextualización de la teología protestante como lugar de cambio del cristianismo en el Pacífico Sur}

Este artículo propone considerar el discurso religioso cristiano del Pacífico Sur contemporáneo, en su versión protestante, como un lugar privilegiado de cambio del campo social. Desde un punto de vista dialógico entre teología, antropología e historia, tres conceptos claves elaborados por tres grandes figuras de la teología protestante contemporánea del Pacífico serán estudiados en primer lugar: la comunidad en la obra del tongano Sione Amanaki Havea, el vanua (el país) en la obra del fiyiano Sevati Tuwere y el aiga (la familia ampliada) en la obra del samoano Ama Amalele Tofaeono. En segundo lugar, se trata de mostrar cómo estos tres conceptos se inscriben en el discurso teológico, sea en cierta continuidad respecto a la teología misionera heredada de finales del siglo XIX y principios del siglo XX, sea al contrario en una transformación entre contextualización crítica y cristianismo de tipo holístico. Luego, se propone una periodización de la teología protestante del Pacífico de los años 1970 a los años 2000, partiendo de una "teología de la identidad" arraigada en la tradición, para llegar a una teología intercultural fundada en el diálogo ecuménico. Por último, la conclusión trata de evaluar el alcance efectivo sobre las instituciones eclesiásticas de este discurso sostenido, como veremos, por intelectuales urbanos ampliamente influenciados por la ideología pan-pacifica de la Pacific Way.

Palabras clave: cristiandad, Oceanía, teología contextual, misiología. 
